\title{
Is the Anatomy of the Inferior Mesenteric Artery Root Different in Patients with a Tortuous Abdominal Aorta?
}

Jia-Hao Zhou

Taipei Tzu Chi Hospital

Hsu-Chao Chang ( $\sim$ hsuchao@gmail.com )

Taipei Tzu Chi General Hospital https://orcid.org/0000-0003-2156-6700

\section{Research article}

Keywords: aorta tortuosity, inferior mesentery artery, computed tomography angiography, angiography

Posted Date: October 1st, 2020

DOI: https://doi.org/10.21203/rs.3.rs-84052/v1

License: (c) (1) This work is licensed under a Creative Commons Attribution 4.0 International License.

Read Full License 


\section{Abstract}

Background: Catheterization of the inferior mesentery artery (IMA) during angiography can sometimes be difficult due to anatomic variations. Our study aimed to evaluate the relationship between abdominal aortic tortuosity (AAT) and the anatomy of the IMA root.

Methods: In this retrospective study, a total of 45 patients were selected and 3 groups were divided using a qualitative method by visual estimation of vessel tortuosity. A quantitative method was performed by recording the patient age, tortuosity index, and features of the IMA root anatomy, including orifice level, vertical diameter, clockwise angle, and angulation. Pearson correlation coefficients $(p<0.05)$ were calculated to analyse the strength of the linear association between tortuosity and other variables.

Results: The AAT index was significantly associated with age. When the abdominal aorta was tortuous, the IMA root was more likely to be distorted toward the convex side, with a larger angulation between the IMA root and the abdominal aorta.

Conclusions: Our study provides information which could help clinicians plan endovascular interventions of IMA. When cannulation of the IMA appears to be difficult, a rotation of tip of the catheter to the convex side of the abdominal aorta and a less sharp looping of the catheter may be attempted.

\section{Background}

Catheterisation of the inferior mesentery artery (IMA) during angiography can sometimes be difficult due to variations in the patients' anatomy. A previous study using three-dimensional CT to evaluate the anatomy of IMA showed that it arises from the aorta approximately $7 \mathrm{~cm}$ below the origin of the superior mesentery artery (SMA). It is usually at the level of $L 3$, and is a relatively straight vessel arising from left side of aorta [1]. Another study evaluating the positional relationships of the inferior mesenteric artery using the coeliac trunk and SMA as landmarks in 32 cadavers revealed that the site of branching of the IMA can be inferred to some extent from the celiac axis and superior mesenteric artery [2].

In this study, we aimed to investigate whether the anatomy of the IMA root was different in patients with increased abdominal aortic tortuosity (AAT).

\section{Methods}

This retrospective clinical study was approved by the Institutional Review Board.

\section{Patient population}

A total of 45 patients who underwent abdominal CT angiography (CTA) between January 2017 and December 2019 were selected and divided into three groups. The groups were composed of 15 patients without AAT (group A), 15 patients with significant AAT and a leftward convexity (group B), and 15 
patients with significant AAT and a rightward convexity (group C). Patients were grouped using a qualitative method involving visual estimation of vessel tortuosity. None of the patients had any history of vascular diseases (e.g. abdominal aortic aneurysm, aortic surgery, or severe atheromatous plaque).

\section{Quantitative image analysis}

Patient age, tortuosity index, and features of the IMA root anatomy, including orifice level, vertical diameter, clockwise angle, and angulation were recorded. The imaging study was performed in Philips CT workstation (Intellispace Portal v7.0.1, Philips Healthcare) with automatic batch creation. The tortuosity index of the abdominal aorta was calculated as the ratio of the centreline to the shortest distance from the diaphragm level to the aortic bifurcation. The clockwise angle was measured in a clockwise fashion from 12:00 to the IMA orifice in the tangential plane of the abdominal aorta. The angle of the IMA was measured at the intersection of the centreline of the abdominal aorta with the IMA root (Fig. 1).

\section{Statistical analysis}

Data were analysed using SPSS Statistics 23 software (IBM Corp, Armonk, NY), and all numerical values were reported as mean \pm standard deviation. Independent-sample $t$-test was used for comparison between groups. A $p$-value of $<0.05$ was considered statistically significant. Pearson correlation coefficients $(p<0.05)$ were calculated to analyse the strength of the linear association between tortuosity and other variables.

\section{Results}

Groups $A, B$, and $C$ each included 15 patients, with mean ages of $48.87 \pm 15.40$ years, $74.13 \pm 8.73$ years, and $73.67 \pm 11.67$ years, respectively. The AAT indices of groups $A, B$, and $C$, were $1.007 \pm 0.002,1.089 \pm$ 0.026 , and $1.119 \pm 0.066$, respectively. The IMA orifice originated at $L 2$ for $2 \%(1 / 45)$ of patients, $L 3$ for $80 \%(36 / 45)$ of patients, and L4 for $18 \%$ (8/45) of patients. Vertical diameters were $3.63 \pm 0.76 \mathrm{~mm}, 3.48$ $\pm 0.83 \mathrm{~mm}$, and $3.18 \pm 0.75 \mathrm{~mm}$ in groups $A, B$, and $C$, respectively. Clockwise angles were $23.92 \pm 9.12$ degrees, $26.65 \pm 14.86$ degrees, and $16.28 \pm 7.02$ degrees in groups $A, B$, and $C$, respectively. The angulations were $38.27 \pm 8.78$ degrees, $46.68 \pm 11.32$ degrees, and $52.85 \pm 8.77$ degrees in groups $A, B$, and $\mathrm{C}$, respectively (Table 1 ). 
Table 1

Comparison of demographic characteristics and vascular features obtained from computed tomography angiography in study groups.

\begin{tabular}{|c|c|c|c|c|c|c|}
\hline & Normal $^{a}$ & Leftward ${ }^{b}$ & Rightward ${ }^{b}$ & \multicolumn{3}{|c|}{$p$-value ${ }^{d}$} \\
\hline & (group A) & (group B) & (group C) & \multirow{2}{*}{$\begin{array}{l}\text { A. vs } \\
\text { B. }\end{array}$} & \multirow{2}{*}{$\begin{array}{l}\text { A. vs } \\
\text { C. }\end{array}$} & \multirow{2}{*}{$\begin{array}{l}\text { B. vs } \\
\text { C. }\end{array}$} \\
\hline & $n=15$ & $n=15$ & $\mathrm{n}=15$ & & & \\
\hline Age (years) & $\begin{array}{l}48.87 \pm \\
15.40\end{array}$ & $74.13 \pm 8.73$ & $\begin{array}{l}73.67 \pm \\
11.67\end{array}$ & $<0.01$ & $<0.01$ & 0.90 \\
\hline Sex (male / female) & $5 / 10$ & $2 / 13$ & $8 / 7$ & & & \\
\hline Tortuosity index (TI) & $\begin{array}{l}1.007 \pm \\
0.002\end{array}$ & $\begin{array}{l}1.089 \pm \\
0.026\end{array}$ & $\begin{array}{l}1.119 \pm \\
0.066\end{array}$ & $<0.01$ & $<0.01$ & 0.11 \\
\hline Range for $\mathrm{TI}$ & $\begin{array}{l}1.003- \\
1.011\end{array}$ & $\begin{array}{l}1.055- \\
1.160\end{array}$ & $\begin{array}{l}1.055- \\
1.310\end{array}$ & & & \\
\hline $\begin{array}{l}\text { Vertical diameter } \\
(\mathrm{mm})\end{array}$ & $3.63 \pm 0.76$ & $3.48 \pm 0.83$ & $3.18 \pm 0.75$ & 0.62 & 0.11 & 0.30 \\
\hline $\begin{array}{l}\text { Clockwise angle } \\
\text { (degree) }\end{array}$ & $23.92 \pm 9.12$ & $\begin{array}{l}26.65 \pm \\
14.86\end{array}$ & $16.28 \pm 7.02$ & 0.55 & 0.02 & 0.02 \\
\hline $\begin{array}{l}\text { Angulation } \\
\text { (degree) }\end{array}$ & $38.27 \pm 8.78$ & $\begin{array}{l}46.68 \pm \\
11.32\end{array}$ & $52.85 \pm 8.77$ & 0.03 & $<0.01$ & 0.11 \\
\hline \multicolumn{7}{|c|}{ Data are presented as mean \pm standard deviation. } \\
\hline \multicolumn{7}{|c|}{ a Patients in group $A$ had no abdominal aortic tortuosity. } \\
\hline \multicolumn{7}{|c|}{${ }^{b}$ Patients in group B had abdominal aortic tortuosity with a leftward convexity. } \\
\hline \multicolumn{7}{|c|}{${ }^{c}$ Patients in group $C$ had abdominal aortic tortuosity with a rightward convexity. } \\
\hline
\end{tabular}

A highly positive correlation was observed between age and the AAT index for both rightward $(r=0.561, p$ $<0.01)$ and leftward $(r=0.732, p<0.01)$ convexities. A moderately positive correlation was identified between the clockwise angle and the AAT index for rightward convexity $(r=0.448, p=0.01)$. However, no statistical correlation was identified for leftward convexity $(r=0.015, p=0.94)$. A highly positive correlation was found between the angulation and the AAT index for rightward convexity $(r=0.545, p<$ $0.01)$, with a moderately positive correlation for leftward convexity $(r=0.393, p=0.03)$ (Table 2). 
Table 2

Pearson correlation coefficients ( $r$ ) for associations between abdominal aortic tortuosity, age, and computed tomography angiography findings in study groups.

\begin{tabular}{|c|c|c|c|c|c|c|}
\hline \multirow[t]{2}{*}{ Variable } & \multicolumn{2}{|c|}{ Tortuosity (A vs B) } & \multicolumn{2}{|c|}{ Tortuosity (A vs C) } & \multicolumn{2}{|c|}{ Tortuosity (B vs C) } \\
\hline & $r$ & $p$-value & $r$ & $p$-value & $r$ & $p$-value \\
\hline Age & $0.732^{\mathrm{a}}$ & $<0.01$ & $0.561^{a}$ & $<0.01$ & 0.156 & 0.41 \\
\hline Vertical diameter & 0.103 & 0.50 & 0.262 & 0.16 & 0.144 & 0.45 \\
\hline Clockwise angle & 0.015 & 0.94 & $0.448^{b}$ & 0.01 & 0.308 & 0.10 \\
\hline Angulation & $0.393^{b}$ & 0.03 & $0.545^{a}$ & $<0.01$ & 0.174 & 0.36 \\
\hline \multicolumn{7}{|c|}{ a Highly positive correlation } \\
\hline \multicolumn{7}{|c|}{${ }^{b}$ Moderately positive correlation. } \\
\hline $\begin{array}{l}\text { Abbreviations: } A, ~ \\
\text { convexity). }\end{array}$ & A (witho & tuosity); & oup $B(\mid \epsilon$ & ard conve & $C$, grou & 'rightward \\
\hline
\end{tabular}

\section{Discussion}

In this study, we demonstrated that the AAT index was significantly related to patient age. The IMA root was more likely to be distorted toward the convex side, with a larger angulation between the IMA root and the abdominal aorta when the abdominal aorta was tortuous.

The IMA is responsible for supplying blood to the distal third of the transverse colon, the descending colon, sigmoid colon, and upper rectum. It is a relatively straight vessel with several branches, all of which arise from the left side, typically between the L2 and L4 vertebral bodies [3, 4]. In our study, the majority $(80 \%)$ of IMA orifices originated at L3, while $18 \%$ originated at L4. Only one patient $(2 \%)$ had an IMA orifice originating at L2.

Previous studies have demonstrated a strong association between vessel tortuosity and mechanical factors, including blood pressure, blood flow axial tension, and structural changes to vessel walls [5]. The influence of age on aortic tortuosity, however, remains controversial [6]. In recent studies, the tortuosity and length of the thoracic aorta were reported to be moderately to significantly associated with age in the ascending and descending segments $[7,8]$. In our study, the AAT index was also shown to be significantly associated with age.

The tortuosity of an artery can be measured using both qualitative and quantitative methods; however, the visual estimation of a vessel's tortuosity is most often used in clinical settings [9]. In our experience, the AAT can be estimated using radiography. Abdominal computed tomography or a guidewire tract during angiography studies may be use if calcified plaques are present in the abdominal aorta. 
In our study, a right counter clockwise rotation of the IMA orifice was shown to have a moderately positive correlation with AAT and a rightward convexity. This finding may be explained by recognising parallels in patients with scoliosis. Using a common computed tomography (CT) method for measuring vertebral rotation in patients with scoliosis, it has been shown that the vertebrae are distorted toward the convex side, with spinous processes deviated to the concave side during disease progression (Fig. 2) [10, 11]. Similarly, a clockwise rotation of the IMA orifice was observed in patients with AAT and with a leftward convexity (Fig. 3). However, there was no statistically significant association between these two variables. We hypothesised two possible reasons for this finding. First, we observed less severe AAT with leftward versus rightward convexities (tortuosity index: 1.089 vs.1.119, respectively). Additionally, IMA orifices were not always located at the curvature sites.

A moderately to highly positive correlation was also identified between angulation and AAT. A kyphotic curvature of the abdominal aorta was observed in both groups $B$ and $C$ except in cases with lateral deviation. During a fixed peritoneal attachment, a wider intersection angle can be achieved with greater kyphotic curvatures (Fig. 4).

There were two limitations in our study. First, the tortuosity index can change depending on the two end points selected. In our study, we chose the diaphragm and the aortic bifurcation as our two end points because they were easily identifiable on imaging studies. Second, as described above, intersecting points are not always the greatest curvature site of the selected vessel, especially when there are more than two consecutive curvatures.

\section{Conclusion}

In our study, the AAT index was shown to be significantly related to patient age. When the abdominal aorta was tortuous, the IMA root tends to be distorted toward the convex side, with a larger angulation between the IMA root and the abdominal aorta. Our study provides information which could help clinicians plan endovascular interventions of IMA. When cannulation of the IMA appears to be difficult, a rotation of tip of the catheter to the convex side of the abdominal aorta and a less sharp looping of the catheter may be attempted.

\section{List Of Abbreviations}

AAT, abdominal aorta tortuosity; ACS, automatic current selection; CL, centreline; CTA, computed tomography angiography; IMA, inferior mesenteric artery; SMA, superior mesenteric artery; SD, shortest distance.

\section{Declarations}

Ethics approval and consent to participate: 
The retrospective study was approved by the Institutional Review Board (Ethic committee of the Taipei Tzu Chi Hospital) with a waiver of informed consent (IRB NO.:09-X-086).

Consent for publication:

Not applicable

Availability of data and materials:

The dataset supporting the conclusions of this article is included within the article and its additional files.

Competing interests:

The authors declare that they have no competing interests.

Funding:

This study was supported by the grant of Taipei Tzu Chi Hospital, Buddhist Tzu Chi Medical Foundation (TCRD-TPE-109-58).

Authors' contributions:

$\mathrm{JHZ}$ - designed the study, data acquisition, image post-processing, analysis of the data and the statistical evaluation. HCC - designed the study, writing of manuscript, corresponding author. All authors read and approved the final manuscript.

Acknowledgements:

This study was supported by the grant of Taipei Tzu Chi Hospital, Buddhist Tzu Chi Medical Foundation (TCRD-TPE-109-58).

\section{References}

1. Horton KM, Fishman EK. Volume-rendered 3D CT of the Mesenteric Vasculature: Normal Anatomy, Anatomic Variants, and Pathologic Conditions. 2002;22(1):161-172.

2. Nakayama Y, Hayashi S, Takeuchi K, Kawata S, Qu N, Itoh M. Positional relationships of abdominal aortic branches for contrast radiography of the inferior mesenteric artery using the coeliac trunk and superior mesenteric artery as landmarks. Okajimas Folia Anatomica Japonica. 2017;93:139-145.

3. Kahn P, Abrams HL. Inferior mesenteric arterial patterns. Radiology. 1964;82:429-42.

4. Chou CK, Mak CW, Hou CC, Chang JM, Tzeng WS. CT of the mesenteric vascular anatomy. Abdom Imaging. 1997;22:477-82.

5. Han HC. Twisted blood vessels: symptoms, etiology and biomechanical mechanisms. J Vasc Res. 2012;49:185-97. 
6. Ciuricâ S, Lopez-Sublet M, Loeys BL, Radhouani I, Natarajan N, Vikkula M, Maas AHEM, Adlam D, Persu A. Arterial tortuosity. Hypertension. 2019;73:951-60.

7. Akin I, Nienaber CA. Age-dependent aortic elongation: a new predictor for type A aortic dissection? Heart. 2018;104:1729-30.

8. Belvroy VM, de Beaufort HWL, van Herwaarden JA, Bismuth J, Moll FL, Trimarchi S. Tortuosity of the descending thoracic aorta: normal values by age. PLoS One. 2019;14:e0215549.

9. Metz H, Bannister RG, Murray-Leslie RM, Bull JWD, Marshall J. Kinking of the internal carotid artery in relation to cerebrovascular disease. Lancet. 1961;277: 424-6.

10. Aaro S, Dahlborn M, Svensson L. Estimation of vertebral rotation in structural scoliosis by computer tomography. Acta Radiologica. Diagnosis. 1978;19:990-2.

11. Lam GC, Hill DL, Le LH, Raso JV, and Lou EH. Vertebral rotation measurement: a summary and comparison of common radiographic and CT methods. Scoliosis. 2008;3:16.

\section{Figures}
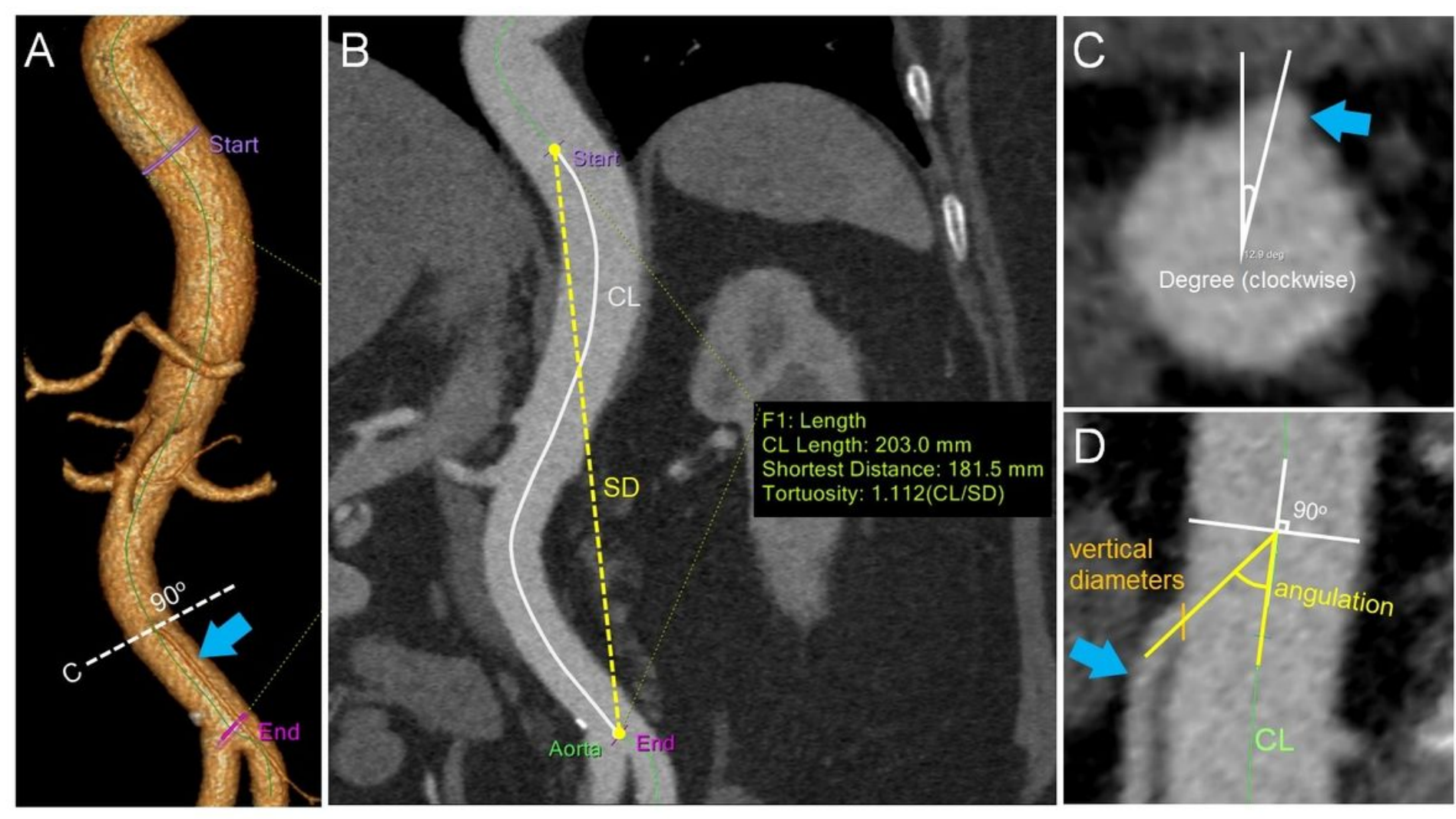

\section{Figure 1}

Qualitative methods (blue arrows point to the inferior mesenteric artery (IMA). (a) The tortuosity index of the abdominal aorta was identified from the level of the diaphragm to the aortic bifurcation and (b) was calculated as the ratio of the centreline (CL) to the shortest distance (SD). (c) The clockwise angle was measured in a clockwise fashion from 12:00 to the IMA orifice in the tangential plane of the abdominal 
aorta. (d) The angulation of the IMA was measured by the intersection of the centreline of the abdominal aorta with the IMA root. The vertical diameter was also measured on this image.
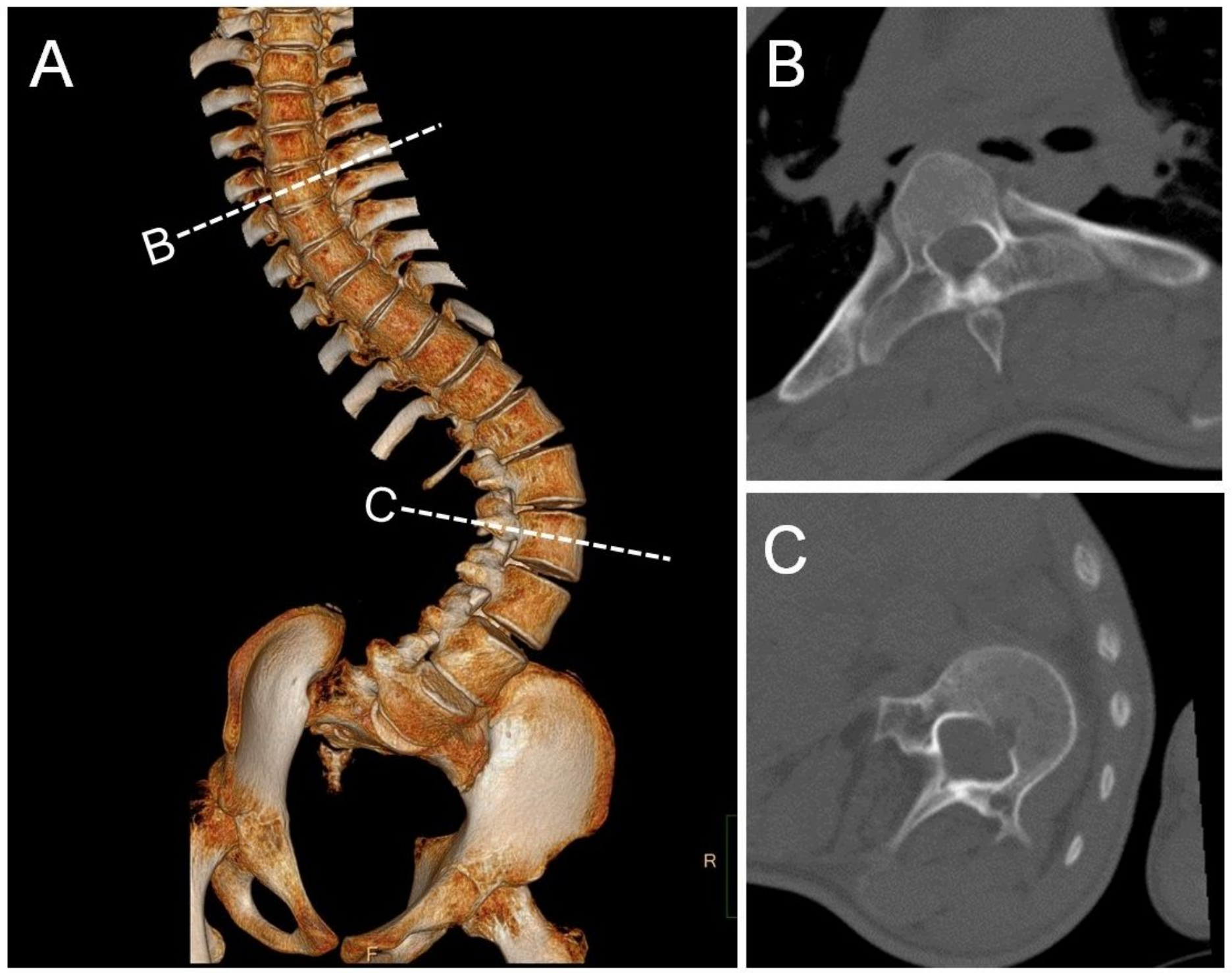

$\mathrm{R}$

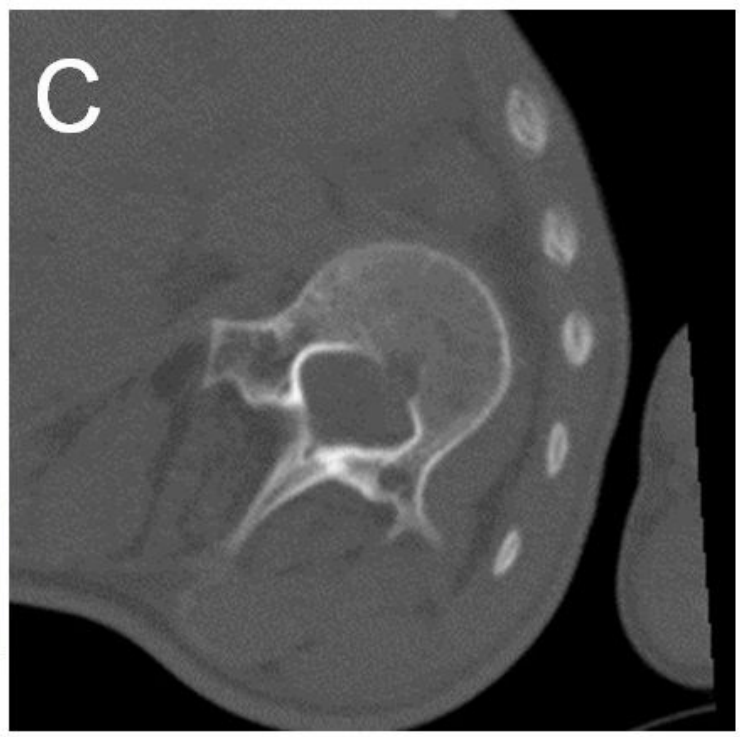

Figure 2

Vertebral rotation in scoliosis. (a) A three-direction volume-rendering image showing two tangential planes of the spine. (b) The vertebra is distorted toward the right side in an area with a rightward convexity of the spine and (c) distorted toward the left side at an area with a leftward convexity of the spine. 


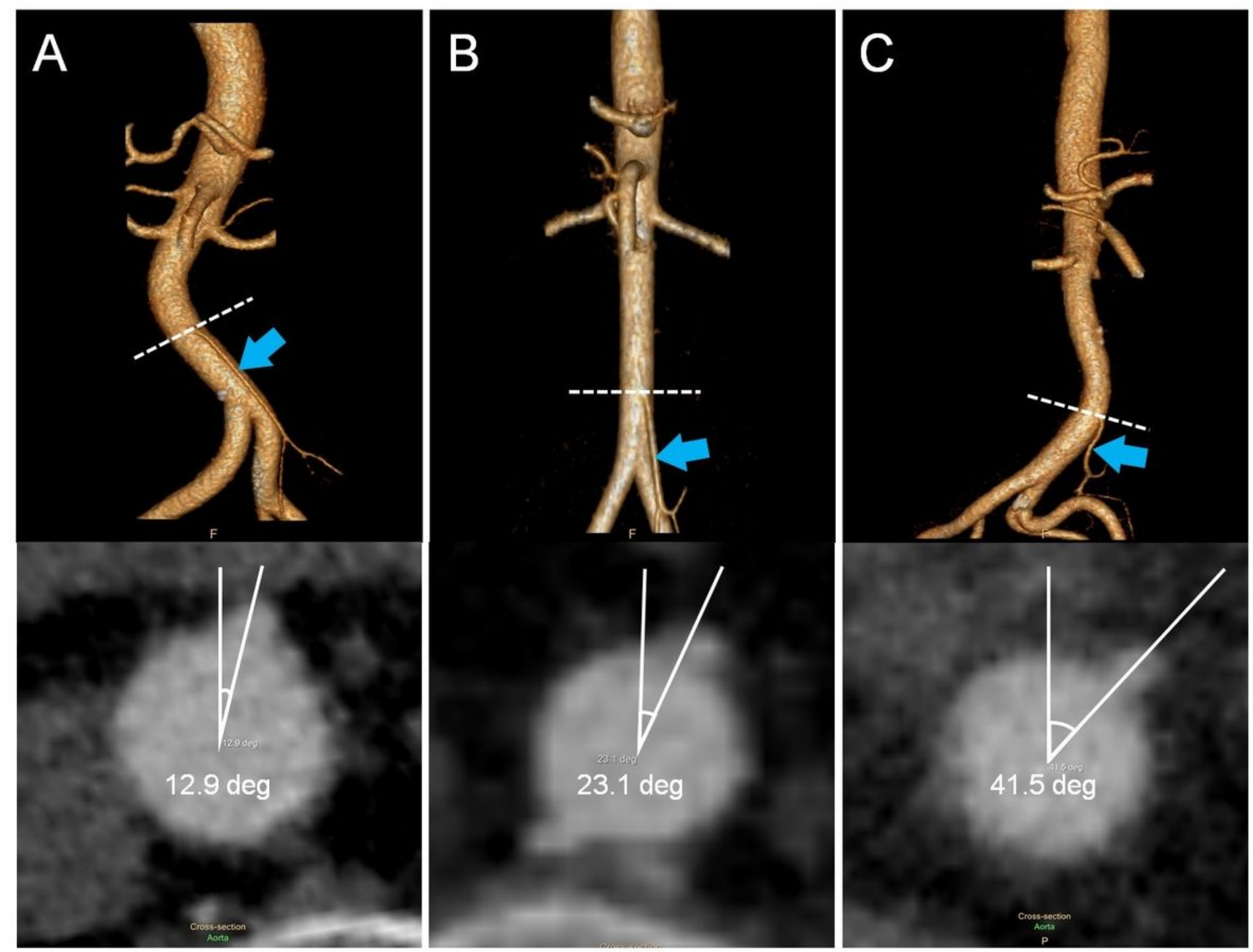

\section{Figure 3}

Clockwise angles were measured with the abdominal aorta having (a) a rightward convexity, (b) neutral position, and (c) leftward convexity. Blue arrows point to the inferior mesenteric artery. 

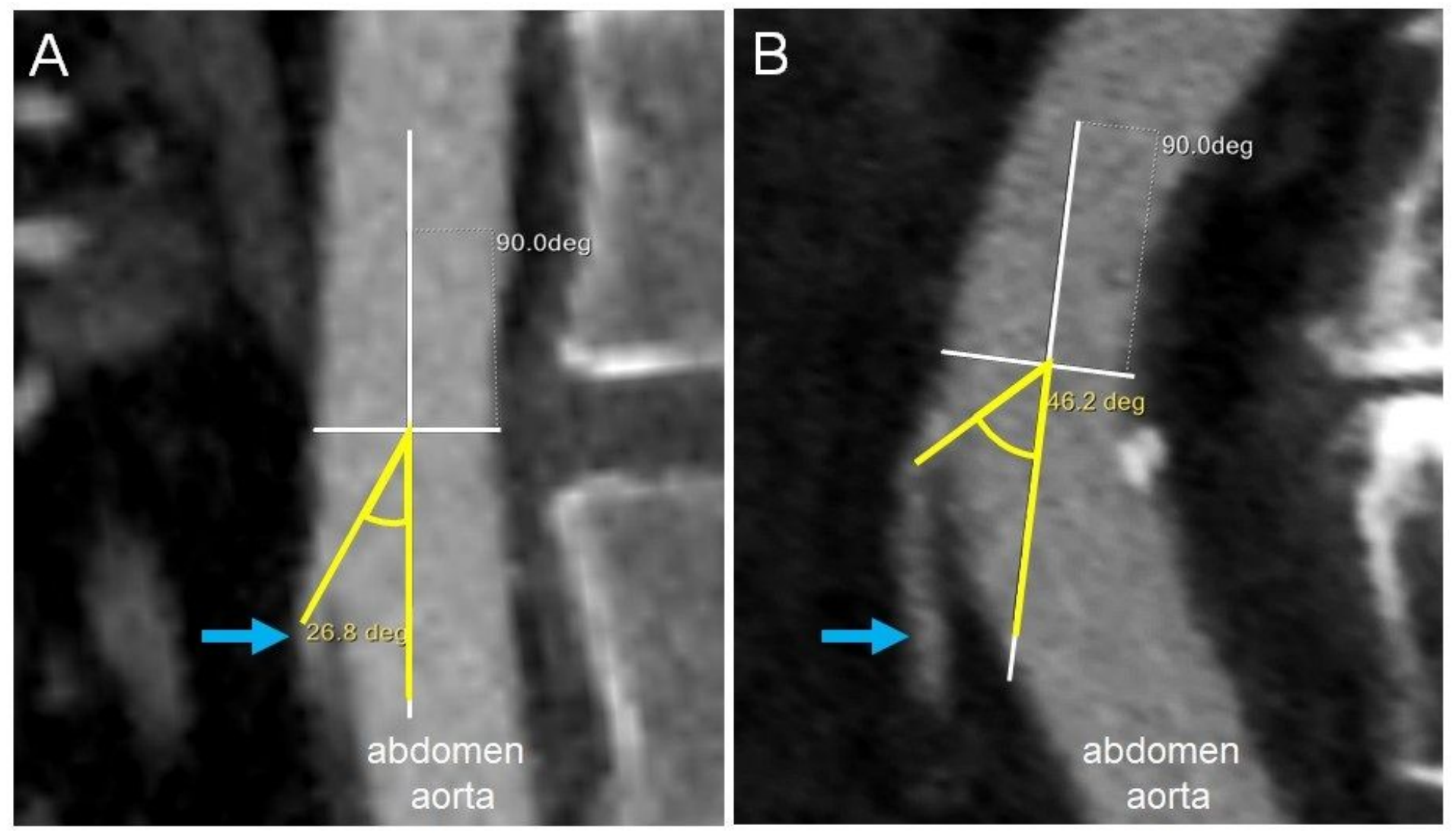

\section{Figure 4}

Angulations were measured in patients (a) without abdominal aortic tortuosity (AAT) and (b) with significant AAT. Note the lordotic curvature of the abdominal aorta identified in (b). Blue arrows point to the inferior mesenteric artery. 